\title{
Relations entre perceptions des producteurs et surfertilisation en maraichage urbain et péri urbain au Bénin
}

\author{
Séraphin Capo ATIDEGLA ${ }^{1 *}$, Wilfrid BONOU ${ }^{1}$ et Euloge Kossi AGBOSSOU ${ }^{1,2}$ \\ ${ }^{1}$ Faculté des Sciences Agronomiques (FSA), 01 BP 526, Cotonou, Bénin. \\ ${ }^{2}$ Institut National de l'Eau (INE), 01 BP 526, Cotonou, Bénin. \\ *Auteur correspondant,E-mail : atideglaser@gmail.com
}

\section{RESUME}

En raison du faible niveau de fertilité des terres du littoral et à l'insuffisance des superficies exploitables due surtout à l'accélération de l'urbanisation, les maraîchers de Grand-Popo ont fait l'option de l'intensification agricole par la surfertilisation. La présente étude a pour but d'évaluer les relations entre deux perceptions de ces maraîchers et la surfertilisation des cultures avec les engrais minéraux et la fiente de volaille. Les données ont été collectées à travers des observations directes et des entretiens sur la base de questionnaires auprès de 100 maraîchers opérant sur les différents sites. Le traitement statistique des données a été fait à l'aide du modèle de régression binaire logit. L'analyse de la première perception a montré que l'option est déterminée par le nombre d'années d'expérience. Quant à la seconde, elle a révélé que la connaissance préalable de certaines maladies détermine la connaissance ou non des risques sanitaires liés à une utilisation intense des engrais minéraux et de la fiente de volaille. La prise en compte de ces facteurs « expérience » et « connaissance » devient une nécessité dans la mise en place des stratégies devant permettre aux maraîchers d'appréhender les risques sanitaires liés à une utilisation intense desdits intrants.

(C) 2017 International Formulae Group. All rights reserved.

Mots clés : Littoral, fertilité, légumes, engrais minéraux, fiente de volaille, risques sanitaires

\section{Relations between farmers perceptions and over dose fertilization in urban and peri urban market gardening in Benin}

\section{ABSTRACT}

By taking into account the low fertility level of the littoral soils and the lack of cropping lands due to urbanization growing, Grand-Popo farmers opt for agricultural intensification by using over dose fertilization. The present study aims to assess the relations between two perceptions of Grand-Popo market gardeners and over dose fertilization of vegetables with mineral fertilizers and poultry manure. Data were collected through field observations and discussion based on questionnaires addressed to 100 market gardeners of the different production sites. The statistic treatment of the data was done with the model of logit binary regression. The analysis of the first perception showed that the choice is determined by the number of years of experience. The analysis of the second perception revealed that the previous knowledge of some diseases determines the knowledge or not of the sanitary risks linked to an intense utilization of chemical fertilizers and poultry manure. Taking into account of the two factors "experience" and "knowledge" becomes a necessity in the 
implementation of strategies which should allow market gardeners to find out the sanitary risks linked to an intense use of those inputs.

(C) 2017 International Formulae Group. All rights reserved.

Key words: Littoral, fertility, vegetables, chemical fertilizers, poultry manure, sanitary risks

\section{INTRODUCTION}

Depuis plus de deux décennies, le maraîchage urbain et péri urbain est devenu l'une des activités phares de la Commune de Grand-Popo. Ces cinq dernières, cette activité est réalisée sur un total de plus de 500 ha dans la Commune de Grand-Popo (CeRPA Mono-Couffo, 2010). Mais, au regard des nombreuses contraintes qui tendent de s'opposer à son développement, dont notamment la croissance démographique, l'agriculture urbaine et péri urbaine constitue l'une des préoccupations majeures en Afrique Subsaharienne (Temple et al., 2004). Au titre de cette agriculture urbaine, la production maraîchère occupe une place de choix au Bénin du fait de sa contribution à la sécurité alimentaire et à la réduction du taux de chômage dans les milieux urbains, périurbains et surtout en bordure du littoral (Hounkpodoté et Tossou, 2001). C'est le cas de la Commune de Grand-Popo où les cultures maraîchères cultivées sont dominées par les légumes feuilles (grande morelle), les légumes racines (carotte), les légumes fruits (tomate, piments) et les légumes à bulbes (oignon). Selon Assogba-Komlan et al. (2007), dans le Sud-Bénin, les légumes feuilles occupent la deuxième place derrière la tomate et sont consommés par $62,5 \%$ de la population béninoise. En dépit de l'infertilité des sols du littoral et de la pression foncière, l'essor de cette activité dans la commune de Grand-Popo se justifie entre autres par l'accès facile à l'eau souterraine (la nappe phréatique étant située entre 3 et $6 \mathrm{~m}$ de la surface du sol). Mais il faut reconnaître que ce essor cadre bien avec les travaux de Forster et al. (2010) selon qui les besoins alimentaires deviendront plus croissants en Asie et en Afrique au cours des prochaines décennies Toutefois, s'il est vrai que l'irrigation permet d'accroître les rendements, il n'en demeure pas moins que l'intensification par l'utilisation abusive des engrais minéraux et organiques ait des répercussions sur la qualité et la conservation des écosystèmes côtiers et de leurs ressources. En effet, pour satisfaire une demande sans cesse croissante en dépit des nombreuses contraintes dont celles liées à l'infertilité des sols et à la pression foncière, les producteurs ont adopté des pratiques culturales qui sont loin de garantir la qualité sanitaire des légumes produits. Il s'agit entre autres de la surexploitation des terres et de la surfertilisation organique et/ou minérale. En effet, des doses d'engrais organiques (fientes de volaille) de 20 à 50 t/ha et minéraux de 0,8 à $1,2 \mathrm{t} / \mathrm{ha}$ sont fréquemment appliquées. Ces doses sont très supérieures à celles recommandées ( $20 \mathrm{t} / \mathrm{ha}$ de fiente de volaille et $400 \mathrm{~kg} / \mathrm{ha}$ d'engrais minéraux) par l'Institut National pour la Recherche Agricole du Bénin (INRAB, 2001). Cette pratique d'intensification présente des risques sanitaires de contamination sévère du milieu et des légumes par les biocontaminants et polluants (germes fécaux, métaux lourds, nitrates, nitrites) (Midmore et al., 2003 ; De Bon et al., 2009; Atidégla, 2011). Contrairement à ces problèmes souvent très documentés, les informations relatives aux facteurs déterminant la pratique de la surdose au cours de la fertilisation et ceux liés à la connaissance des risques sanitaires par les maraîchers sont marginales. La présente étude vise donc à évaluer les facteurs influençant le respect ou non de la dose de $20 \mathrm{t} / \mathrm{ha}$ de fiente de volaille et ceux déterminant la connaissance ou non des risques sanitaires liés à une intense utilisation des engrais minéraux et de la fiente de volaille dans les périmètres maraîchers urbains et péri urbains de GrandPopo. 


\section{MATERIEL ET METHODES}

Zone d'étude

Située dans le Département du Mono, en bordure de la côte Atlantique, la Commune de Grand-Popo est entre $6^{\circ} 15^{\prime}$ et $6^{\circ} 25^{\prime}$ de latitude Nord et entre $1^{\circ} 40^{\prime}$ et $1^{\circ} 55$ de longitude Est, avec une superficie de $240 \mathrm{~km}^{2}$ (CeRPA Mono-Couffo, 2010). Son altitude moyenne est de $5 \mathrm{~m}$ au-dessus du niveau de la mer. Elle s'étend sur une longueur de $35 \mathrm{~km}$ de côte et abrite une population de 57636 habitants (INSAE, 2013). Comme toute la région côtière, elle jouit d'un climat de type sub-équatorial, caractérisé par de faibles variations de température (moyenne annuelle de $27,4{ }^{\circ} \mathrm{C}$ ) et une pluviométrie bimodale : une grande saison sèche de novembre à avril, une grande saison pluvieuse d'avril à juillet, une petite saison sèche d'août à septembre et une petite saison pluvieuse de septembre à octobre. La moyenne pluviométrique annuelle est de $882,1 \mathrm{~mm}$. La zone d'étude fait partie de l'ensemble géomorphologique de la zone $\mathrm{du}$ littoral qui est un domaine très complexe à cause de la diversité et du dynamisme des éléments qu'il associe : la mer, les deltas, les lagunes, les lacs, les marécages et les cordons littoraux.

\section{Choix des villages}

Une enquête exploratoire a permis de retenir, les Arrondissements de Grand-Popo et d'Agoué, disposant d'exploitations maraîchères urbaines et péri urbaines fonctionnelles toute l'année. En effet, les maraîchers y pratiquent souvent quatre campagnes agricoles chaque année, ce qui est favorable à la conduite de nos recherches. Le choix des villages a été fait suivant les critères ci-après : disposer d'au moins dix (10) exploitations maraîchères, avoir les exploitations de part et d'autre de la Route Nationale Inter Etats 1 (RNIE 1) CotonouLomè sur une distance de $600 \mathrm{~m}$ au plus, du bord de ladite route, disposer d'au moins dix maraîchers exerçant chacun sur une superficie supérieure ou égale à 0,5 ha. A défaut d'une liste complète de toutes les exploitations et du fait que les exploitations concernées par l'étude sont toutes celles appartenant au village retenu, il a été procédé au choix des exploitations fonctionnelles par village.

\section{Echantillonnage des enquêtés}

L'unité statistique d'enquête est le maraîcher dans son exploitation. Quel que soit le système d'exhaure (traditionnel ou amélioré) utilisé par le maraîcher (arrosoir, motopompe ou pompe électrique), les critères appliqués pour opérer le choix sont : disposer d'au moins 50 planches, avoir déjà eu à conduire au moins deux campagnes de cultures, être présent dans l'exploitation au cours du passage de l'enquêteur, se faire recenser une seule fois. Ceci concerne les maraîchers qui disposent de plus d'une exploitation dans la zone d'étude et qui ne conduisent pas eux-mêmes les activités sur les sites.

\section{Collecte des données}

Des entretiens ont été organisés avec des focus groupes de maraîchers de tous les sites maraîchers de Grand-Popo. Cette phase a permis de recenser de façon informelle les contraintes auxquelles sont confrontés les maraîchers et d'identifier leurs perceptions sur les pratiques de fertilisation des sols avec les engrais (minéraux et organiques) et les risques sanitaires y afférents. Ces informations ont servi d'indicateurs de référence pour l'élaboration du questionnaire administré lors de la phase de collecte des données spécifiques à l'étude. Cette phase d'enquête proprement dite a été réalisée auprès d'un échantillon de 100 maraîchers ayant rempli les critères de choix dans les Arrondissements de Grand-Popo et d'Agoué.

\section{Traitement des données}

Les données relatives aux perceptions des populations de la zone d'étude par rapport aux risques sanitaires liés à une utilisation intense des fertilisants ont été traitées selon les deux axes ci-après :

- l'analyse des facteurs qui influencent le respect ou non de la dose de la fiente de 
volaille effectuée à l'aide du modèle de régression binaire logit (Collett, 1991),

- l'analyse selon le même modèle des facteurs qui influencent la connaissance ou non des risques sanitaires liés à une utilisation intense des engrais minéraux et de la fiente de volaille.

Le modèle de régression binaire logit a été utilisé pour modéliser les deux analyses.

Le modèle de régression binaire fait partie d'une famille de modèles appelés modèles linéaires généralisés. Un modèle linéaire généralisé est défini par une fonction de lien notée g qui met en relation la composante aléatoire (vecteur $\mathrm{Y}$ ) et la composante systématique (matrice $\mathrm{X}$ et vecteur des coefficients). La linéarité du modèle dépend $\mathrm{du}$ choix de $\mathrm{g}$, donc de la transformation des valeurs de Y. Il existe plusieurs fonctions de lien : logit, probit, log-log en particulier. D'après Collett (1991), la fonction logit est la plus employée, essentiellement à cause de sa simplicité d'usage.

Si $\mathrm{f}(\mathrm{x})$ représente la probabilité pour un individu d'appartenir au groupe $i$ des individus codés 1 , probabilité notée $\pi_{i}$ aussi appelée probabilité a posteriori ou probabilité de l'événement $i$, alors la forme du modèle logistique s'écrit:

$f(x)=\pi_{i}=\frac{\exp ^{\left(\beta_{0}+\beta_{1} x_{i}\right)}}{1+\exp ^{\left(\beta_{0}+\beta_{1} x_{i}\right)}}$

$\beta_{0}$ et $\beta_{1}$ sont les coefficients du modèle. La transformation de $\pi_{i}$ utilisée s'appelle la transformation logit. Elle est donnée par la relation suivante :

$\log \left(\pi_{i}\right)=\log \left(\frac{\pi_{i}}{1-\pi_{i}}\right)=g=\beta_{0}+\beta_{1} x_{i}$

où $\log$ représente le logarithme népérien.

Lorsque $p$ variables explicatives sont considérées sachant que $j$ est l'indice des variables et $i$ celui des individus, la fonction $g$ s'écrit :

$g=\beta_{0}+\beta_{1} x_{1 i}+\beta_{2 i} x_{2 i}+\cdots+\beta_{p i} x_{p i}$
Analyse des facteurs influençant le respect ou non de la dose de 20 t/ha de fiente de volaille

Appliqué aux données de cette étude, le modèle devient: RESPFIENTE $=\beta_{0}+$ $\beta_{1}$ SEXE $+\beta_{2}$ NIVEAU $+\beta_{3}$ EXPE + $\beta_{4} S U P T O T+\beta_{5}$ CREDIT $+\beta_{6} P O L L U+$ $\beta_{7} M A U V Q U A L+\beta_{8} S A N T E+$ $\beta_{9} A C C R O I R D T+\beta_{10} B N E Q U A L$. Ce modèle suppose une variable dépendante latente à deux modalités. En effet, le respect de la dose de fiente (RESPFIENTE) représente la variable dépendante (Y). Elle (RESPFIENTE) prend la valeur 1 pour un producteur, si ce dernier a respecté la dose de fiente et la valeur 0 si non. Les variables explicatives introduites sont au nombre de 10 et sont indiquées dans le Tableau 1.

La recherche documentaire et l'enquête de terrain sur l'amendement des sols ont montré que la présence des femmes affecte positivement l'adoption des engrais organiques et le respect de la dose préconisée. En effet, les femmes de la zone respectent le plus les consignes techniques. Ainsi, le sexe du chef exploitation est défini par la variable (SEXE). Le niveau d'étude du maraîcher (NIVEAU) semble avoir une influence dans le respect de la dose, de même que son ancienneté qui est positivement et fortement corrélé avec le nombre d'années d'expérience dans le maraîchage. En effet, les producteurs qui ont une longue expérience (EXPE) dans la pratique des cultures maraîchères utilisent fortement les engrais organiques et minéraux pour lutter contre l'infertilité des sols. Cette variable prend la valeur 1 si le nombre d'années d'expériences est $\geq 3$ et 0 , si c'est le contraire. La superficie totale emblavée pour le maraîchage (SUPTOT) qui pourrait avoir un effet positif sur l'utilisation de la fiente de volaille est également prise en compte dans le modèle. Cette variable prend la valeur 1 si la superficie totale emblavée est $\geq 0,2$ ha et 0 , si c'est le contraire. Pour tester l'hypothèse que l'accès au crédit financier permet aux producteurs d'utiliser la fiente de volaille dont les coûts d'acquisition (achat et transport) sont très élevés, la source principale de 
financement de la lutte contre l'infertilité des sols (CREDIT) est incluse dans le modèle. La conscience de la plupart des maraîchers par rapport à la pollution des eaux, sols et produits maraîchers (POLLU) qui découle de la surfertilisation est également prise en compte dans le modèle. Dans la même dynamique des impacts négatifs liés à la surdose en matière de fertilisation, l'hypothèse mauvaise qualité des légumes a été testée (MAUVQUAL) dans le modèle. Puisque ces deux dernières variables sont positivement et fortement corrélées avec la santé des consommateurs et des producteurs, la santé (SANTE) semble avoir une influence dans le respect de la dose. Cependant, du fait que l'application de la fiente de volaille permet d'obtenir de bons rendements pour la production et également une bonne qualité des produits maraîchers, les variables (ACCROIRDT) et (BNEQUAL) ont été intégrées dans le modèle.

Analyse des facteurs influençant la connaissance ou non des risques sanitaires liés à une utilisation intense des engrais minéraux et de la fiente de volaille

L'analyse des facteurs qui influencent la connaissance ou non des risques sanitaires liés à une intense utilisation des fertilisants a été effectuée en utilisant le même modèle de régression logistique binaire. Ainsi, appliqué aux données de l'étude, le modèle devient: CONRISQSANIT $=\beta_{0}+\beta_{1}$ SEXE $+\beta_{2}$ NIVEAU $+\beta_{3}$ CONSOMEAUX $+\beta_{4}$ CONMALADIE + $\beta_{5}$ CONCAUSE où, la variable dépendante (CONRISQSANIT) est égale à 1 , si l'enquêté connaît au moins un risque sanitaire lié à l'intense utilisation des fertilisants, et prend la valeur 0 dans le cas contraire. Les variables explicatives introduites dans le modèle sont au nombre de 5 et sont présentées dans le Tableau 2.

En dehors des variables «SEXE» et «NIVEAU » utilisés dans le premier modèle, les variables « CONSOMEAUX », «CONMALADIE » et « CONCAUSE » y ont été intégrées, afin de tester respectivement les hypothèses selon lesquelles la consommation des eaux d'arrosage, la connaissance des maladies et la connaissance des causes des maladies pourraient avoir des effets positifs sur la connaissance ou non des risques sanitaires liés à une intense utilisation des engrais minéraux et organiques.

Tableau 1 : Variables explicatives introduites dans le modèle RESPFIENTE.

\begin{tabular}{lll}
\hline $\begin{array}{l}\text { Variables } \\
\text { explicatives }\end{array}$ & Signification et niveau de mesure & Signes attendus \\
\hline SEXE & Sexe (Masculin $=1$, Féminin $=0$ ) & + \\
\hline NIVEAU & Niveau d'étude (Niveau $\geq$ secondaire $=1$ Si non $=0)$ & + \\
\hline EXPE & $\begin{array}{l}\text { Expérience en nombre d'années dans le maraîchage } \\
\text { (Plus de } 3 \text { ans }=1, \text { moins de } 3 \text { ans }=0)\end{array}$ & + \\
\hline SUP & Superficie emblavée (Superficie emblavée $\geq 0,2 \mathrm{ha}=1, \leq 0,2 \mathrm{ha}=0)$ & + \\
\hline CREDIT & Accès au crédit pour l'achat des engrais (Oui $=1$ et Non $=0)$ & + \\
\hline POLLU & Pollution des eaux, sols et produits maraîchers $($ Oui $=1$ et Non $=0)$ & + \\
\hline MAUVQUAL & Mauvaise qualité des produits (Oui $=1$ et Non $=0)$ & + \\
\hline ACCROIRDT & Accroissement du rendement (Oui $=1$ et Non $=0)$ & + \\
\hline BNEQUAL & Bonne qualité des produits maraîchers $($ Oui $=1$ et Non $=0)$ & + \\
\hline SANTE & Risques sanitaires (Oui $=1$ et Non $=0)$ & + \\
\hline
\end{tabular}


Tableau 2 : Variables explicatives introduites dans le modèle CONRISQSANIT.

\begin{tabular}{|c|c|c|}
\hline $\begin{array}{l}\text { Variables } \\
\text { explicatives }\end{array}$ & Signification et niveau de mesure & $\begin{array}{c}\text { Signes } \\
\text { attendus }\end{array}$ \\
\hline SEXE & Sexe $($ Masculin $=1$, Féminin $=0)$ & + \\
\hline NIVEAU & Niveau d'étude $($ Niveau $\geq$ secondaire $=1$ Si non $=0$ ) & + \\
\hline CONMEAUX & Consommation d'eau (Puits ou forage artificiel=1, SONEB ou AEP = 0) & + \\
\hline CONMALADIE & $\begin{array}{l}\text { Connaissance de certaines maladies clés (Connais au moins deux des dix } \\
\text { maladies suivantes comme risque sanitaire lié à la pollution des eaux, sols } \\
\text { et légumes) \{(1) Intoxication ou gastro-entérites ; (2) Fièvre typhoïde ; (3) } \\
\text { Fatigue, Maux de tête (4) Choléra ; (5) Troubles respiratoires ou toux; } \\
\text { (6) Dermatoses ; (7) Tension ; (8) Diabète ; (9) Cancer ; (10) Maux de } \\
\text { reins }\}=1 \\
\text { Si non }=0\end{array}$ & + \\
\hline CONCAUSE & $\begin{array}{l}\text { Connaissance de certaines causes des maladies (Connais au moins une des } \\
\text { causes ci-après : }\{(1) \text { Mauvaise qualité eaux de puits ou forage ; (2) } \\
\text { Mauvaise qualité des produits maraîchers; (3) Manque d'hygiène ou } \\
\text { malpropreté ; (4) Déversement d'ordures et tas d'immondices; (5) } \\
\text { défécation en plein air par hommes et animaux }\}=1 \\
\text { Si non }=0\end{array}$ & + \\
\hline
\end{tabular}

\section{RESULTATS}

\section{Nécessité de la fertilisation des sols de Grand-Popo}

Dans toute la zone d'étude, la pauvreté des sols est bien connue des maraîchers que de l'ensemble de la population locale. Plusieurs facteurs sont à l'origine de la pauvreté des sols de Grand-Popo parmi lesquels, les facteurs naturels (pédologie, hydrographie, climat, pluviométrie,) humains (explosion démographique, pression foncière, etc.), économiques (recherche de revenus à travers les cultures intensives de produits maraîchers, accroissement des cheptels bovins, caprins et porcins), agronomiques (mauvaises pratiques culturales). En effet, la pluviosité caractérisée par sa variabilité spatiale et temporelle reste difficile ou impossible à changer alors qu'en revanche, le sol peut subir des transformations en terme d'amélioration ou de dégradation en fonction de son utilisation ou des moyens qu'on y emploie. Pour ce faire, les maraîchers de Grand-Popo ont fait l'option d'accentuer leurs actions en faveur de l'amélioration de la qualité fertilisante du sol d'où la nécessité de la fertilisation.

D'après les enquêtés, fertiliser c'est satisfaire les besoins des sols et plantes en nutriments à travers les apports d'engrais organiques (fèces et déchets des animaux, débris de végétaux), engrais minéraux notamment le NPK (quelle que soit la formule), l'urée et à la mise en jachère des sols après plusieurs années de mise en valeur. Pour eux, les sols sableux de la zone du littoral ne sont favorables qu'aux cocotiers et autres plantes et arbres sauvages et les utiliser pour les besoins agricoles impose des amendements à l'exploitant. Pour une agriculture intensive en maraîchage urbain et périurbain à Grand-Popo, cet amendement 
doit être permanent et continu car, un champ qui est fréquemment fumé est en permanence «noir » à l'image des champs qui sont à proximité des concessions parce qu'ils ne perdent jamais de leur fertilité.

En effet, avant le démarrage du maraîchage à grande échelle à Grand-Popo, les champs de case qui étaient pratiqués par les ménages tout autour des concessions ne recevaient pas d'engrais minéraux, ni d'engrais organiques; mais leurs sols avaient servi de réceptacles des déchets ménagers qui tenaient lieu de fumier. Lesdits champs de case recevaient essentiellement les cultures du gombo (hibiscus esculentus) et du crin-crin (corchorus olitorius). Aujourd'hui, c'est l'ère de l'utilisation intense des engrais minéraux et de la fiente de volaille pour amender les périmètres maraîchers urbains et péri urbains servant à la production diversifiée de légumes. Elle peut avoir des raisons multiples dont notamment :

$>$ la tendance à surdoser pour s'assurer une marge de sécurité en matière de fertilité du sol ;

$>$ la difficulté à évaluer les réserves du sol qui dépendent des apports précédents, du volume de matière organique enfouie, sous forme de racines et de chaumes, et dont la nitrification dépend largement des conditions climatiques ;

> la recherche d'un rendement le plus élevé possible qui conduit à augmenter considérablement la fertilisation. En cas d'accident de parcours: aléas climatiques, maladies, erreurs techniques, l'objectif de production ne sera pas atteint et la plante n'utilisera pas tout l'engrais mis à sa disposition.

\section{Appropriation des difficultés liées à l'acquisition des engrais minéraux et organiques par les maraîchers}

Comme la plupart des intrants que sont semences et produits phytosanitaires, les engrais minéraux et organiques ne sont pas disponibles sur place à Grand-Popo et leur acquisition relève d'un vrai parcours de combattant.

\section{Engrais minéraux}

A l'origine, certains agriculteurs avaient perçu l'utilisation de l'engrais coton comme une alternative pour pallier le problème crucial de la baisse de fertilité du sol. En effet, $40 \%$ des plus anciens des maraîchers, totalisant plus de 20 années d'expérience, ont effectivement reconnu avoir utilisé au début des années 1990, les engrais coton NPKSB (Azote-Phosphore-PotasseSoufre-Bore) de formule 1423145 1, parce que c'étaient les seuls dont ils arrivaient à s'en procurer non sans difficultés, dans le département de Couffo à environ 150 kilomètres de Grand-Popo. Cette pratique n'avait duré que quelques années, parce que c'était le seul type d'engrais minéraux disponibles à l'époque pour les producteurs, à un prix raisonnable de $9000 \mathrm{~F} \mathrm{CFA} \mathrm{le} \mathrm{sac} \mathrm{de}$ $50 \mathrm{~kg}$ (à cause de la subvention aux intrants du coton). Au prix de longues périodes de sensibilisation par les agents de l'ex- Centre d'Action Régionale pour le Développement Rural (ex-CARDER Mono) sur les inconvénients que cela comporte à savoir, une intoxication sensible des cultures, les maraîchers avaient renoncé aux engrais coton NPKSB, pour adopter les engrais NPK de formules 102020 ou 121118 ou 151515 , indiqués pour les cultures vivrières et/ou maraîchères. Certains des maraîchers qui étaient plus nantis avaient alors commencé par acheter les engrais minéraux urée et NPK de formules 151515 ou 121118 au Togo au prix de $12000 \mathrm{~F}$ CFA le sac de $50 \mathrm{~kg}$. Ils ont étendu leur prospection vers Cotonou et environs où la plupart devaient se ravitailler en ces types d'engrais à $14500 \mathrm{~F}$ CFA le sac de $50 \mathrm{~kg}$. Quelques commerçants saisirent l'opportunité pour livrer directement à GrandPopo les engrais minéraux aux maraîchers qui 
le désirent à un prix variant entre 15000 et 16000 F CFA. Mais, le comble, c'est qu'il arrive fréquemment des périodes où, ni l'engrais NPK, ni l'urée ne sont disponibles sur les marchés béninois et/ou togolais ou en nombre très insuffisant pour couvrir les besoins du moment. Dans ces conditions, la plupart des producteurs qui pratiquent la combinaison engrais minéraux et engrais organiques, se rabattent sur la fiente de volaille et /ou la bouse de vache. D'après les résultats d'enquête, il importe de souligner qu'au cours d'un cycle, $100 \%$ des maraîchers appliquent les engrais minéraux selon au moins trois fois la dose recommandée (400 $\mathrm{kg} / \mathrm{ha}$ ) par les structures de recherche du Bénin.

\section{Engrais organiques}

$\mathrm{La}$ fumure organique consiste en l'apport de déjections animales (fiente de volaille et bouse de vache). La mise en œuvre de cette opération dépend du budget de chaque producteur, et à l'image des engrais minéraux, les déjections animales sont achetées en dehors de Grand-Popo, car les producteurs ne sont pas les responsables des élevages fournisseurs qui n'existent d'ailleurs pas dans la Commune. La pratique a commencé au même moment que la fertilisation minérale par les maraîchers de la zone d'étude. La bouse de vache était la seconde fumure organique utilisée après quelques essais timides des graines de coton. Au début des années 2000, la bouse de vache s'achetait à Comè et ses environs (dans un rayon de 35 à $60 \mathrm{~km}$ ) au prix moyen variant de 500 à $800 \mathrm{~F}$ CFA le sac de $50 \mathrm{~kg}$ selon qu'on se trouve chez l'éleveur ou sur les sites à Grand-Popo. Sa disponibilité n'était pas toujours garantie dans les élevages fournisseurs à cause de la demande sans cesse croissante. Pour s'affranchir de cette difficulté, certains maraîchers allaient s'approvisionner à Anécho au Togo à un prix relativement plus cher. Les mêmes difficultés étaient ressenties au niveau de la fiente de volaille dont le prix d'acquisition était relativement plus cher (700 à 1300 F CFA le sac de $50 \mathrm{~kg}$ ). Il convient de souligner que, les élevages de volaille qui ravitaillent les maraîchers de Grand-Popo n'existent que dans les environs de Ouidah, Athiémé, Lokossa et Cotonou c'est-à-dire dans un rayon de 100 à $140 \mathrm{~km}$ des sites. Le commerce des engrais organiques est beaucoup plus organisé que celui des engrais minéraux à Grand-Popo. Il est pratiqué par quelques éleveurs qui viennent eux-mêmes directement livrer sur les sites. Certains producteurs à court d'argent ou n'ayant pas bénéficié de crédit de campagne s'arrangent auprès des éleveurs commerçants pour acheter à crédit la fiente de volaille ou la bouse de vache à récupérer à la vente des légumes. Eu égard à la cherté des engrais, les producteurs ont très souvent recours aux institutions de micro finance qui leur octroient des crédits de campagne pour l'acquisition de ces intrants.

\section{Facteurs socio-économiques de la fertilisation des sols}

Les producteurs de la zone d'étude ont une bonne connaissance des engrais organiques et minéraux qui améliorent la qualité de ces sols ainsi que ceux qui contribuent à sa pollution. Il en est de même des impacts positifs et négatifs qu'ont ces fertilisants sur les produits maraîchers. A force de les pratiquer, ils ont fini par reconnaître les engrais qui sont plus indispensables que d'autres ainsi que les risques sanitaires qui découlent à moyen ou long termes de l'utilisation de ces engrais en agriculture intensive.

Les pratiques paysannes de fertilisation des sols sont déterminées par une série de paramètres visant à permettre au producteur d'atteindre les objectifs liés à la forte productivité souhaitée, la sécurité alimentaire de son ménage et la maximisation du profit. Ainsi, pour comprendre les raisons qui 
justifient la prise de décision chez le producteur, il est nécessaire de combiner les différents facteurs susceptibles d'influencer positivement ou négativement sa prise de décision. De plus, la connaissance du poids de chacun des facteurs qui influencent la prise de décision permettra d'identifier les indicateurs clés sur lesquels devra se baser la recherche pour l'amélioration qualitative et quantitative de la production maraîchère.

\section{Respect ou non de la dose de 20 t de fiente de volaille à l'hectare}

La statistique de Hosmer et Lemeshow $(1>0,05)$ montre qu'il y a une description adéquate des observations par le modèle (Tableau 3). Globalement, le modèle bivarié appliqué est significatif (Prob. $=0,001$ ). L'hypothèse principale d'un lien entre le respect ou non de la dose de fiente de volaille et certains facteurs (variables explicatives) est donc confirmée. Le modèle de régression permet de classifier correctement environ 79 $\%$ des observations ; c'est-à-dire que dans $79 \%$ de cas, ce modèle prédit correctement le comportement du producteur ("respect ou non" de la dose de fiente). Cependant, le modèle est peu performant avec un coefficient de détermination relativement faible $(0,225)$; ce qui signifie que seulement $22,5 \%$ de la variabilité du respect ou non de la dose de fiente de volaille par les producteurs est expliqué par les variabilités des variables explicatives.

Le modèle estimé identifie une seule variable pertinente qui explique l'attitude des producteurs quant au respect ou non de la dose de fiente. Il s'agit de la variable Nombre d'années d'expérience (EXPE). Le coefficient de cette variable est significatif à $1 \%$. Cette variable est positivement corrélée avec la probabilité de respect de la dose de fiente de volaille. En effet, plus un producteur a une longue expérience en termes de nombre d'années dans le maraîchage, plus il respecte la dose de fiente à appliquer et vice versa.
Connaissance ou non des risques sanitaires liés à une intense utilisation des engrais minéraux et la fiente de volaille

La statistique de Hosmer et Lemeshow $(1>0,05)$ montre qu'il y a une description adéquate des observations par le modèle (Tableau 4). Le modèle est significatif (Prob. $<0,001)$; ce qui permet de confirmer qu'il existe un lien entre la connaissance des risques sanitaires liés aux activités de fertilisation des sols et les facteurs explicatifs (variables explicatives) ci-dessus mentionnés. Le modèle de régression permet de classifier correctement $84,3 \%$ des observations. En d'autres termes, ce pourcentage indique que dans $84 \%$ de cas, ce modèle prédit correctement le comportement du maraîcher en ce qui concerne la "connaissance ou non" des risques sanitaires liés à son activité.

De plus, le modèle peut être qualifié de performant avec un coefficient de détermination relativement élevé $(0,58)$; ce qui signifie que $58 \%$ de la variabilité de la connaissance des risques sanitaires liés aux activités de maraîchage par les producteurs est expliqué par les variabilités des variables explicatives considérées.

Cependant, le modèle identifie une seule variable pertinente qui explique la connaissance des risques sanitaires liés aux activités de maraîchage par les maraîchers. Il s'agit de la variable Connaissance de certaines maladies (CONMALADIE). Le coefficient de cette variable est positif et significatif à $0,1 \%$. En effet, seule la prévalence de certaines maladies telles que : la fièvre typhoïde, l'intoxication ou gastroentérite, le choléra, les arthroses ou courbatures, la toux ou les troubles respiratoires, le diabète, le cancer, constitue une preuve de connaissance des risques sanitaires par les maraîchers vivant dans la zone d'étude. 
S. C. ATIDEGLA et al. / Int. J. Biol. Chem. Sci. 11(5): 2106-2118, 2017

Tableau 3 : Variables explicatives à coefficients significatifs et autres statistiques du modèle RESPFIENTE.

\begin{tabular}{|c|c|c|c|c|c|c|}
\hline $\begin{array}{l}\text { Variables retenues par le } \\
\text { modèle }\end{array}$ & B & S.E. & Wald & Df & Sig. & $\operatorname{Exp}(B)$ \\
\hline EXPE & 1,509 & 0,558 & 7,311 & 1 & 0,007 & 4,523 \\
\hline \multicolumn{7}{|c|}{ Autres statistiques } \\
\hline Nombre d'observations & \multicolumn{6}{|c|}{100} \\
\hline $\begin{array}{l}\text { Test de Hosmer et } \\
\text { Lemeshow }\end{array}$ & \multicolumn{6}{|c|}{1,000} \\
\hline $\operatorname{chi}^{2}(3)$ & \multicolumn{6}{|c|}{15,625} \\
\hline $\begin{array}{l}\text { Signification globale du } \\
\text { modèle }\end{array}$ & \multicolumn{6}{|c|}{0,001} \\
\hline -2Log pseudolikelihood & \multicolumn{6}{|c|}{87,167} \\
\hline $\begin{array}{l}\text { Pouvoir de prédiction } \\
\text { correcte }\end{array}$ & \multicolumn{6}{|c|}{79,0} \\
\hline Pseudo $\mathrm{R}^{2}$ & \multicolumn{6}{|c|}{0,225} \\
\hline
\end{tabular}

Tableau 4 : Variables explicatives à coefficients significatifs et autres statistiques du modèle CONRISQSANIT.

\begin{tabular}{lcccccc}
\hline Variables retenue par le modèle & B & S.E. & Wald & Df & Sig. & Exp (B) \\
\hline CONMALADIE & 1,684 & 0,214 & 62,150 & 1 & 0,000 & 5,385 \\
\hline \multicolumn{7}{l}{ Autres statistiques } \\
\hline Nombre d'observations & \multicolumn{7}{c}{166} \\
\hline Test de Hosmer et Lemeshow & \multicolumn{7}{c}{1,000} \\
\hline chi $^{2}(3)$ & 144,099 \\
\hline Signification globale du modèle & 0,000 \\
\hline -2Log pseudolikelihood & $-72,050$ \\
\hline Pouvoir de prédiction correcte & 84,3 \\
\hline Pseudo $\mathrm{R}^{2}$ & \multicolumn{7}{c}{0,580} \\
\hline
\end{tabular}




\section{DISCUSSION}

Conséquences de l'intense utilisation des engrais minéraux et la fiente de volaille sur la qualité des légumes

D'après plusieurs sources concordantes de la littérature, l'objectif de la fertilisation est d'apporter aux sols les éléments minéraux et organiques dont ils ont besoin pour assurer une bonne productivité aux cultures (Fall et al., 2000 ; Galantini et al., 2006 ; Björn et al., 2010). Toutefois, un apport excessif d'engrais azoté contribue à la détérioration de la qualité des légumes produits (Assogba-Komlan, 2007). Les pratiques souvent observées sont le non-respect des doses et des fréquences d'application, l'utilisation d'engrais minéraux de qualité douteuse sans précision de composition chimique et d'engrais inappropriés, comme ce fut le cas des engrais coton NPKSB. Conformément aux résultats du modèle 1 , le non respect de la dose pour la fiente de volaille est de règle, sauf chez les producteurs les plus expérimentés ayant déjà réuni de longues années de pratique. En effet, pour ces derniers, au fil des années et tenant compte des résultats obtenus par rapport à l'historique de la fertilisation des sites d'une part, et conscients des conséquences fâcheuses liées à une surfertilisation des légumes, décident librement de la dose appropriée à appliquer pour la fiente de volaille. Cette catégorie de maraîchers $(21 \%)$ respecte la dose recommandée de 20 tonnes de fiente de volaille à l'hectare. Puisque la fertilisation consiste à améliorer les propriétés physiques, chimiques et biologiques du sol afin de favoriser les conditions de croissance des plantes et d'obtenir ainsi des rendements plus élevés et des produits de meilleure qualité (De Bon et al., 2009 ), une surfertilisation conduit non seulement à la détérioration de la qualité de la plante produite, mais aussi contribue à la dégradation de l'environnement. Les méfaits de la surfertilisation ont été également dénoncés par la plupart des enquêtés qui reconnaissent presque tous les risques sanitaires attachés à cette pratique à travers les résultats du modèle 2. En termes clairs, ce qui préoccupe les producteurs, c'est l'augmentation de revenus car le maraîchage urbain et péri urbain à Grand-Popo n'est qu'une activité de survie. Ceci est conforme aux travaux de Cissé et al., (2002) à Ouagadougou et se comprend aisément tant qu'aucune autre alternative ne s'offre concrètement pour la frange de la population qui l'exerce.

\section{Conscience des maraîchers de la zone d'étude}

Au terme des investigations et eu égard aux résultats issus de l'application des modèles étudiés «RESPFIENTE» et "CONRISQSANIT», il ressort que les habitants de la zone d'étude sont conscients des risques sanitaires liés à une intense utilisation d'engrais (chimiques et organiques) par les maraîchers. De surcroît, les diverses informations qui caractérisent le maraîchage et les maraîchers de Grand-Popo, laissent entrevoir déjà le but pour lequel l'activité est pratiquée. Mais comme avant tout, les maraîchers ne destinent leur production que pour la vente, certains vendent alors, consciemment ou inconsciemment, des produits infectés aux consommateurs rien que pour se faire de l'argent. Au prix d'une forte productivité et de rendements meilleurs, gages d'une amélioration de revenus, certains producteurs ne se sentent pas gênés, malgré leur connaissance des risques, de continuer l'activité avec les mauvaises pratiques agricoles (Moustier et al., 2004 ; Keys et al., 2005). C'est pourquoi, très peu d'entre eux respectent la dose de $20 \mathrm{t} / \mathrm{ha}$ pour la fiente de volaille à cause de leur ancienneté dans le domaine. Sans doute que ceux qui respectent cette dose ont reconnu les arrières effets des fertilisants antérieurs et préfèrent ne pas gaspiller en abusant. Pour la plupart, ils n'ont cessé de déclarer que, c'est pour se faire de l'argent à travers la commercialisation qu'ils produisent et qu'ils n'avaient même pas une habitude alimentaire de ces produits. Ils disent 
être conscients de la situation mais qu'ils ne changeront de comportement que lorsque des solutions alternatives leur seront proposées. La pollution des eaux de la nappe est bien connue d'eux mais disent-ils, ils préfèrent boire cette eau que de crever de soif. Au moins $40 \%$ ont reconnu quelques cas de contamination à travers les nombreuses maladies hydriques qui sont enregistrées sur les périmètres. Les non maraîchers qui ont cependant double casquette parce qu'exerçant pour la plupart, le maraîchage comme activité secondaire, partagent les mêmes points de vue que les maraîchers exclusifs. Dans l'ensemble et comme l'ont proposé Kenmogne et al., (2010), les enquêtés tout en reconnaissant les dangers qui guettent la non durabilité de leurs systèmes agricoles et la dégradation très poussée de l'environnement souhaitent l'appui des autorités locales et des services techniques de l'agriculture pour les aider à sortir de cette impasse.

\section{Conclusion}

Pour réduire les risques sanitaires en direction des producteurs et consommateurs, des interventions doivent porter principalement sur les deux facteurs " expérience» et «connaissance» des maladies. Pour le premier facteur, elles porteront sur la sensibilisation, la formation et l'encadrement technique des producteurs tandis que pour le second, elles porteront également sur la sensibilisation basée sur des études de cas et les aspects comme le revers (ou effets indésirables) de la surfertilisation. C'est pourquoi, tout en visant des rendements élevés qui permettent d'obtenir des revenus substantiels, le producteur ne doit pas perdre de vue qu'il s'intoxique lui-même à travers l'eau qu'il consomme et que lui-même, consciemment ou inconsciemment a eu à polluer, même s'il ne consomme pas les légumes. Tenir compte de ces aspects conduirait à surmonter ces difficultés et de trouver selon Smith, (2002) des approches de solution aux problèmes que posent plusieurs auteurs sur la qualité des légumes. En effet, comme l'ont souligné Agossou et al., (2001), tout se passe comme si les consommateurs locaux n'avaient pas besoin de consommer des produits de qualité.

\section{CONFLIT D'INTERETS}

Les auteurs déclarent qu'il n'y a aucun conflit d'intérêts.

\section{CONTRIBUTIONS DES AUTEURS}

SCA a procédé à l'enquête de terrain et à la rédaction de l'article. WB s'est occupé du traitement statistique et EKA a procédé à la correction de tout le document.

\section{REMERCIEMENTS}

La présente étude n'aurait pas pu être réalisée sans l'assistance financière $d u$ Laboratoire d'Hydraulique et de Maîtrise de l'Eau de la Faculté des Sciences Agronomiques (FSA) de l'Université d'Abomey-Calavi (UAC), à travers le Projet NPT 145 .

\section{REFERENCES}

Agossou G, Ahouansou T, Aly D, AssogbaKomlan F. 2001. Etude sur la promotion de la filière des cultures maraîchères au Bénin. Rapport principal (version provisoire), MAEP, Bénin. $75 \mathrm{p}$.

Assogba-Komlan F, Achigan E, Sikirou R, Boko A, Adje C, Ahle V, Vodouhè R, Assa A. 2007. Pratiques culturales et teneur en éléments anti nutritionnels (nitrates et pesticides) du Solanum macrocarpum au sud du Bénin. African Journal of Food, Agriculture, Nutrition and Development, 7: 1-21.

Atidégla CS. 2006. Atouts et contraintes des modes d'irrigation dans les exploitations urbaines et péri urbaines de la Commune de Grand-Popo. Mémoire de DEA, EDP/FLASH, Université d'AbomeyCalavi (UAC), Bénin, 78 p.

Atidégla CS. 2011. Effets des différentes doses d'engrais minéraux et de la fiente de volaille sur l'accumulation de biocontaminants et polluants (germes fécaux, composés azotés et phosphorés, métaux lourds) dans les eaux, les sols et les légumes de Grand-Popo au Bénin. Thèse de doctorat unique, EDP/FLASH, 
Université d'Abomey-Calavi (UAC), Bénin, 314 p.

Azontondé A, Verheye W. 1986. Pédologie et aptitude des terres. Etude de Factibilité pour le Développement Rural Intégré de la Province du Mono, Centre National d'Agro-Pédologie, Rapport d'étude, Abomey-Calavi, Bénin, 89 p.

Björn K, Olaf C, Kurt-Jürgen H. 2010. Modelling nitrogen cycles of farming systems as basis of site- and farmspecific nitrogen management. Agric. Ecosyst. Environ., 135(1-2): 70-80. https://doi.org/10.1016/j.agee.2009.08.01 4

CeRPA (Centre Régional pour la Promotion Agricole des Départements MonoCouffo), 2010. Plan de campagne 2009. 2010, Lokossa, 146 p.

Cissé G, Kientgam, Ouedraogo B, Tanner M. 2002. Développement du maraîchage autour des eaux de barrage à Ouagadougou : quels sont les risques sanitaires à prendre en compte ? Cahiers Agricultures, 11(1): 31-38.

Collett D. 1991. Modelling Binary Data. Scientific Book for Assessment: London \& Hall; 45 p.

De Bon H, Parrot L, Moustier P. 2009. Sustainable urban agriculture in developing countries. A review. Agro. Sustain. Dev., 30: 21-32.

Fall S-T, Aminata N, Fall A-S, Cissé I, Badiane A-N, Fall $\mathrm{CH}$, Ba Diao M. 2000. Intégration horticulture - élevage dans les systèmes agricoles urbains de la zone des Niayes (Sénégal). Bulletin de $l^{\prime} A P A D$ [En ligne], $19 \mid 2000$, URL : http://apad.revues.org/444

Forster D, Menzi H, Schertenleib R, Lennartz B. 2010. Assessing patterns of nitrogen management in peri urban agriculture of Hanoi, Vietnam. Urban Agriculture Magazine, 23: 5-16, www.ruaf.org

Galantini J, Rosell R. 2006. Long-term fertilization effects on soil organic matter quality and dynamics under different production systems in semiarid Pampean soils. Soils an Tillage Res., 87: 1, 72-79

INRAB (Institut de Recherches Agricoles du Bénin), 2001. Fiche technique de production de la variété améliorée de tomate dans les périmètres maraîchers du Bénin. INRAB, Cotonou.

INSAE. 2013. Quatrième recensement général de la population et de l'habitation. Synthèse des résultats. INSAE, 35p.

Kenmogne GRK, Rosillon F, Mpakam HG, Nono A. 2010. Enjeux sanitaires, socioéconomiques et environnementaux liés à la réutilisation des eaux usées dans le maraîchage urbain: cas du bassin versant de l'Abiergué (YaoundéCameroun). VertigO - la Revue Electronique en Sciences de l'Environnement, 10(2), [En ligne], URL : http://vertigo.revues.org/14976; DOI : 10.4000/vertigo.14976

Keys E, McConnell WJ. 2005. Global change and the intensification of agriculture in the tropics. Globe Environ. Change, Part A. 15: 320-337.

Midmore DJ, Jansen HGP. 2003. Supplying vegetables to Asian cities: is there a case for peri-urban production? Food Policy. 28(2) : 13-27.

Moustier P, Moumbé M, Huat J. 2004. La gestion concertée et durable des filières maraîchères urbaines. In Développement Durable de l'Agriculture Urbaine en Afrique Francophone : Enjeux, Concepts et Méthodes. CIRAD/CRDI (ed): Montpellier, France.

Smith OB. 2002. Overview of urban agriculture and food security in West African cities. In Advances in Croplivestock Integration in West African Cities. ITC-ISRA-IDRC (ed). Printed by Grafisch Bedrijf Ponsen en Looijen B.V.: Wageningen.

Temple L, Moustier P. 2004. Les fonctions et contraintes de l'agriculture péri urbaine de quelques villes africaines (Yaoundé, Cotonou, Dakar). Cah. Agric., 13: 15-22.

Tiamiyou I. 1995. Appui à l'intensification et à la promotion de cultures maraîchères et fruitières au Bénin. Rapport phase III de la consultation en phytotechnique maraîchère du 30 juillet au 12 août 1995 . Projet TCP/BEN/4553 (A), FAO, Bénin, $31 \mathrm{p}$. 